SUSX-TH-01-032

RHCPP 01-03T

hep-th/0108131

July 2001

\title{
STANDARD-LIKE MODELS FROM INTERSECTING D4-BRANES
}

\author{
D.BAILIN " G. G. V. KRANIOTIS [ and A. LOVE \\ \& Centre for Theoretical Physics, \\ University of Sussex, \\ Brighton BN1 9QJ, U.K. \\ \$ Centre for Particle Physics , \\ Royal Holloway and Bedford New College, \\ University of London,Egham, \\ Surrey TW20-0EX, U.K.
}

\begin{abstract}
We construct a one-parameter set of intersecting D4-brane models, with six stacks, that yield the (non-supersymmetric) standard model plus extra vector-like matter. Twisted tadpoles and gauge anomalies are cancelled, and the model contains all of the Yukawa couplings to the tachyonic Higgs doublets that are needed to generate mass terms for the fermions. A string scale in the range $1-10 \mathrm{TeV}$ and a Higgs mass not much greater than the current bound is obtained for certain values of the parameters, consistently with the observed values of the gauge coupling constants.
\end{abstract}

\footnotetext{
${ }^{1}$ D.Bailin@sussex.ac.uk

${ }^{2}$ G.Kraniotis@sussex.ac.uk
} 
The use of D-branes in Type II string theories has led to exciting new possibilities for constructing models with realistic gauge groups and three generations of chiral matter [1]. Since D-branes carry gauge fields localised on their world volumes, an attractive approach is to start from a configuration of D-branes designed to give the standard model gauge group, possibly augmented by additional $U(1)$ factors, and to introduce further D-branes to produce at least the required matter content. The branes are located at an $R^{6} / \mathbb{Z}_{N}$ orbifold fixed point, to ensure $\mathcal{N}=1$ supersymmetry, and the whole system is then embedded in a global compact space, an orbifold or orientifold, for example. Further D-branes and/or Wilson lines as well as anti-D-branes are added to ensure the cancellation of twisted tadpoles and the overall consistency of the theory. This is the "bottom-up" approach pioneered in [2], who also showed that only the $\mathbb{Z}_{3}$ point group can yield three chiral generations of matter. Extra vector-like matter is generic to such models, and it was shown in [3] that this leads to gauge unification at an intermediate string scale between about $10^{10}$ and $10^{12} \mathrm{GeV}$, consistently with the measured value of $\sin ^{2} \theta_{W}\left(m_{Z}\right)$. Despite their attractions these models are not without defects. Specifically, the unavoidable extra D-branes needed to construct the model generate additional gauge groups under which some of the standard model matter is charged. So these gauge groups are not hidden from the observable sector, and the absence of data for such extra gauge groups must be taken as evidence against such models. Further, some of the Yukawa terms needed to generate masses for the quarks and leptons are forbidden by surviving global $U(1)$ symmetries. Also, these global symmetries are spontaneously broken when electroweak symmetry breaking occurs, thereby generating a keV-scale axion [4] which is unambiguously excluded by axion searches.

A different route to the standard model was motivated by Berkooz, Douglas and Leigh's observation [5] that intersecting D-branes can give rise to chiral fermions propagating in the intersection of their world volumes. D-branes at angles afford a T-dual interpretation as D-branes with magnetic flux [6], and based on that paper the stringy methods for what are now called "intersecting brane models" were developed in [7]. This last paper led Aldazabal et al [8, 9] to develop (non-supersymmetric) four-dimensional chiral models from intersecting D4-branes wrapped on 1-cycles of a 2-dimensional torus $T^{2}$ sitting at a singular point in the transverse 4-dimensional space $B$. The local geometry of $B$ near the singularity is modelled as $C^{2} / Z_{N}$. This gives an $\mathcal{N}=2$ supersymmetric gauge sector. Since the models are generically nonsupersymmetric, by virtue of their matter content, solution of the hierarchy problem requires a string scale of not more than $10 \mathrm{TeV}$ or so, although the usual fourdimensional Planck mass can be obtained by making the volume of $B$ large enough. The generic appearance at tree level of scalar tachyons at some intersections allows the interpretation of doublets as electroweak Higgs scalars, although colour-triplet and/or charged singlet tachyons are potentially lethal for theories purporting to be realistic. However, provided that their masses are small compared with the string

3 For other recent work on interesecting brane models, both supersymmetric and nonsupersymmetric, and their phenomenological implications, see reference 10]. 
scale, radiative corrections might overcome this defect. Although these models are non-supersymmetric, they typically contain extra matter, besides that in the standard model, some of which can be light. In particular there are towers of "gonions", massive (fermionic and bosonic), vector-like matter arising from excitations associated with the angle at which the D-branes intersect, and which are charged with respect to the standard model gauge group. There are also Kaluza-Klein and winding-mode towers of gauge bosons which might also exist below the string scale.

All of these features make intersecting brane models susceptible to experimental investigation in the near future, so it is clearly desirable to construct models that are consistent with existing data. A first step in this programme was made in [9] where some three-generation models were constructed using five stacks of D4-branes, each stack wrapping a different 1-cycle of $T^{2}$. Note that two stacks are different if their 1-cycles intersect, or if they are parallel but not overlapping. The stacks sit at a singular point of the transverse space $B$, taken to be $\left(T^{2}\right)^{2} / \mathbb{Z}_{3}$. Our objective in this paper is to construct models which besides satisfying the technical constraints of Ramond-Ramond tadpole and anomaly cancellation also satisfy more of the phenomenological constraints. Specifically, we require that the models allow the (renormalizable) Yukawa couplings needed to generate mass terms for all standard model matter. The gauge couplings in these models are not unified at the string scale; they depend upon the wrapping numbers of the particular stack of D4-branes with which they are associated. So we also require that these values are achieved at a string scale of not more than about $10 \mathrm{TeV}$ using renormalization group equations starting from the measured values of the gauge coupling strengths at the weak scale. We find that these constraints cannot be simultaneously satisfied using at most five stacks of D-branes, but that certain six-stack models can do so.

As in [9], we take the space $B$ transverse to the D4-branes to be $C^{2} / Z_{3}$, where the point group generator $\theta$ of $Z_{3}$ acts on the two complex coordinates of $B$ with twist vector $v=\frac{1}{3}(1,-1)$. We too choose the first stack to have $N_{1}=3 \mathrm{D} 4$-branes and to be sitting at a singular point of $B$ with wrapping numbers $\left(n_{1}, m_{1}\right)=(1,0)$. In general the integers $n_{a}$ and $m_{a}$ count the number of times that the 1-cycle wrapped by the stack $a$ wraps the two basis 1-cycles defining the torus $T^{2}$; there is no loss of generality in this choice of wrapping numbers for the first stack. $\theta$ is embedded in the stack as $\gamma_{\theta}=\alpha^{p} \mathbf{I}_{3}$ which generates a $U(3) \supset S U(3)_{c}$ gauge group. Here $\alpha=e^{2 \pi i / 3}$ and $p=0,1$ or 2. As in [9], we also choose the second stack to generate a $U(2) \supset S U(2)_{L}$ gauge group by taking $N_{2}=2$ D4-branes and embedding $\theta$ as $\gamma_{\theta}=\alpha^{q} \mathbf{I}_{2}$, where $q \neq p \bmod 3$. The wrapping numbers are $\left(n_{2}, 3\right)$, so that the (12) intersection produces $I_{12} \equiv n_{1} m_{2}-n_{2} m_{1}=3$ quark doublets $Q_{L}$. Note that, unlike in the bottom-up approach [2], the number of generations is not determined by the choice of the point group $\mathbb{Z}_{3}$. Since further non-abelian gauge groups are not required, all remaining stacks have just one D4-brane. Without loss of generality these remaining stacks are split into three sets $I, J$ and $K$ having $\gamma_{\theta}=\alpha^{q}, \alpha^{r}$ and $\alpha^{p}$ respectively, where $p, q, r$ are a permutation of $0,1,2$. The wrapping numbers for the stack $i \in I$ are $\left(n_{i}, m_{i}\right)$, and similarly for $j \in J$ and $k \in K$. Table 1 summarizes the assignments. The gauge group resulting from these stacks of branes is at least 


\begin{tabular}{|c|c|c|c|}
\hline \hline Stack $a$ & $N_{a}$ & $\left(n_{a}, m_{a}\right)$ & $\gamma_{\theta}$ \\
\hline \hline 1 & 3 & $(1,0)$ & $\alpha^{p} \mathbf{I}_{3}$ \\
\hline 2 & 2 & $\left(n_{2}, 3\right)$ & $\alpha^{q} \mathbf{I}_{2}$ \\
\hline$i \in I$ & 1 & $\left(n_{i}, m_{i}\right)$ & $\alpha^{q}$ \\
\hline$j \in J$ & 1 & $\left(n_{j}, m_{j}\right)$ & $\alpha^{r}$ \\
\hline$k \in K$ & 1 & $\left(n_{k}, m_{k}\right)$ & $\alpha^{p}$ \\
\hline \hline
\end{tabular}

Table 1: Multiplicities, wrapping numbers and Chan-Paton phases for the D4-brane stacks, $(p \neq q \neq r \neq p)$.

$$
\prod_{a} U\left(N_{a}\right)=U(3) \times U(2) \times \prod_{i} U(1)_{i} \times \prod_{j} U(1)_{j} \times \prod_{k} U(1)_{k}
$$

When $n_{a}$ and $m_{a}$ have a greatest common divisor greater than 1 or one of the $n_{a}$ or $m_{a}$ is zero, there may be additional gauge group factors. The wrapping numbers are constrained by the requirements of twisted tadpole cancellation, which for the system described above gives

$$
\begin{aligned}
3+\sum_{k} n_{k} & =2 n_{2}+\sum_{i} n_{i}=\sum_{j} n_{j} \\
\sum_{k} m_{k} & =6+\sum_{i} m_{i}=\sum_{j} m_{j}
\end{aligned}
$$

The spectrum of these models is generically chiral, so potentially there are gauge anomalies. In general, tadpole cancellation is sufficient to ensure cancellation of the cubic non-abelian anomalies, which in our case arise only for the $S U(3)$ group. Each of the stacks provides a $U(1)$ gauge group, and the weak hypercharge $Y$ is in general a linear combination of the $\mathrm{U}(1)$ charges $Q_{a}$ associated with the $a$ th stack:

$$
Y=c_{1} Q_{1}+c_{2} Q_{2}+\sum_{i} c_{i} Q_{i}+\sum_{j} c_{j} Q_{j}+\sum_{k} c_{k} Q_{k}
$$

For convenience we have suppressed any reference to any extra $U(1)$ factors which have been discussed after eq.(11). These are trivial to incorporate.

So we must also ensure that mixed $U(1)_{Y}-S U(3)_{c}$ and $U(1)_{Y}-S U(2)_{L}$ anomalies are absent, and their cancellation imposes further constraints on the wrapping numbers. To find them we must first fix the coefficients $c_{a}$. The quark doublets $Q_{L}$ that arise from the (12) intersection have $Q_{1}=1, \quad Q_{2}=-1$ and $Q_{a}=0, \quad a \neq 1,2$. Thus we require

$$
c_{1}-c_{2}=\frac{1}{6}
$$

The $(1 i)$ intersections, with $i \in I$, yield colour triplet, $S U(2)$ singlet fermions, which we require to be right-chiral $u$ or $d$ quarks, so

$$
c_{1}-c_{i}=\frac{2}{3} \quad \text { or } \quad-\frac{1}{3}
$$


Similarly for the sets $J, K$. Thus we split the sets $I, J$ and $K$ into $I_{1,2}, J_{1,2}$ and $K_{1,2}$ with

$$
\begin{aligned}
c_{1}-c_{i_{1}, j_{1}, k_{1}} & =\frac{2}{3} \\
c_{1}-c_{i_{2}, j_{2}, k_{2}} & =-\frac{1}{3}
\end{aligned}
$$

where $i_{1} \in I_{1}$ etc. Then

$$
\begin{aligned}
Y & =\left(c_{1}+\frac{1}{3}\right)\left(Q_{1}+Q_{2}+\sum_{i} Q_{i}+\sum_{j} Q_{j}+\sum_{k} Q_{k}\right) \\
& -\left(\frac{1}{3} Q_{1}+\frac{1}{2} Q_{2}+\sum_{i_{1}} Q_{i_{1}}+\sum_{j_{1}} Q_{j_{1}}+\sum_{k_{1}} Q_{k_{1}}\right)
\end{aligned}
$$

It is easy to see that the combination $Q_{1}+Q_{2}+\sum_{i} Q_{i}+\sum_{j} Q_{j}+\sum_{k} Q_{k}$ is zero for all of the chiral fermions, so henceforth we take

$$
Y=-\left(\frac{1}{3} Q_{1}+\frac{1}{2} Q_{2}+\sum_{i_{1}} Q_{i_{1}}+\sum_{j_{1}} Q_{j_{1}}+\sum_{k_{1}} Q_{k_{1}}\right)
$$

Using (3), the cancellation of the mixed $U(1)_{Y}-S U(3)_{c}$ anomaly then requires that the wrapping numbers satisfy

$$
\sum_{i_{1}} m_{i_{1}}+\sum_{j_{1}} m_{j_{1}}-2 \sum_{k_{1}} m_{k_{1}}=-3
$$

Chiral $S U(2)$ doublet fermions arise from the $I_{2 a}=n_{2} m_{a}-3 n_{a}$ intersections between the second stack and the ath stack, and cancellation of the mixed $U(1)_{Y}-S U(2)_{L}$ anomaly requires that the wrapping numbers also satisfy

$$
n_{2}\left(\sum_{i_{1}} m_{i_{1}}+1-\sum_{k_{1}} m_{k_{1}}\right)+\sum_{j_{1}} n_{j_{1}}-2 \sum_{i_{1}} n_{i_{1}}+\sum_{k_{1}} n_{k_{1}}=-1
$$

where we have used eqns.(2),(3) and (11).

All intersecting branes give chiral fermions, but in addition tachyonic scalars arise whenever the Chan-Paton phases of the intersecting branes coincide. Thus we get $S U(2)_{L}$ doublet tachyons at $\left(2 i_{1}\right)$ and $\left(2 i_{2}\right)$ intersections, charged singlet tachyons from $\left(i_{1} i_{2}\right),\left(j_{1} j_{2}\right)$ and $\left(k_{1} k_{2}\right)$ intersections, and $S U(3)_{c}$ colour triplet tachyons from $\left(1 k_{1}\right)$ and $\left(1 k_{2}\right)$ intersections. Doublet tachyons may be interpreted as electroweak Higgs scalars, but the charged singlet and colour triplet tachyons are less welcome. To avoid the latter, the stacks in the sets $K_{1,2}$ must not intersect with those in the first stack, and this requires

$$
m_{k_{1}}=0=m_{k_{2}} \quad \forall k_{1} \in K_{1}, \quad \forall k_{2} \in K_{2}
$$




\begin{tabular}{|c|c|c|c|}
\hline \hline Stack $a$ & $N_{a}$ & $\left(n_{a}, m_{a}\right)$ & $\gamma_{\theta}$ \\
\hline \hline 1 & 3 & $(1,0)$ & $\alpha^{p} \mathbf{I}_{3}$ \\
\hline 2 & 2 & $\left(n_{2}, 3\right)$ & $\alpha^{q} \mathbf{I}_{2}$ \\
\hline $3=i_{1}$ & 1 & $\left(1-n_{2},-3\right)$ & $\alpha^{q}$ \\
\hline $4=i_{2}$ & 1 & $\left(1-n_{2},-3\right)$ & $\alpha^{q}$ \\
\hline $5=j_{1}$ & 1 & $(2,0)$ & $\alpha^{r}$ \\
\hline $6=k_{1}$ & 1 & $(-1,0)$ & $\alpha^{p}$ \\
\hline \hline
\end{tabular}

Table 2: Multiplicities, wrapping numbers and Chan-Paton phases for the six-stack models, $(p \neq q \neq r \neq p)$.

Similarly, to avoid charged singlet tachyons we require

$$
I_{i_{1} i_{2}}=0=I_{j_{1} j_{2}} \quad \forall i_{1} \in I_{1}, \quad \forall i_{2} \in I_{2}, \quad \forall j_{1} \in J_{1}, \quad \forall j_{2} \in J_{2}
$$

As usual, the Yukawa couplings of the Higgs doublets generate fermion mass terms when the electroweak symmetry is spontaneously broken. The existence of Higgs doublets requires that

$$
\sum_{i_{1}}\left|I_{2 i_{1}}\right|+\sum_{i_{2}}\left|I_{2 i_{2}}\right| \neq 0
$$

and we also demand that the model allows the (renormalizable) Yukawa couplings needed to give all quarks masses. This requires that

$$
I_{1} \neq \emptyset \neq I_{2}
$$

Yukawa couplings arise from a disk-shaped world sheet with three open-string vertex operators attached to its boundary. The boundaries of the disk are the relevant D4branes. For example, the insertion of a $Q_{L}$ vertex operator turns an $a=1$ stack D4-brane boundary into a $b=2$ stack D4-brane boundary [11. The allowed Yukawas satisfy selection rules that derive from a $Z_{2}$ symmetry associated with each stack of D4-branes. A state associated with a string between the ath and bth stack of D4 branes is odd under the $a$ th and $b$ th $Z_{2}$ and even under any other $Z_{2}$. It is straightforward to show that models with at most five stacks cannot satisfy all of the above constraints. The only six-stack models that do are parametrized by a single integer $n_{2}$ and are given in Table 2 .

The matter content of these models is easily determined using the results of 8$]$. In general, there are $n_{G}=3$ generations of (massless) chiral fermions plus vector-like fermionic matter and (tachyonic) Higgs doublets of the form

$$
\alpha\left(e^{c}+\bar{e}^{c}\right)+\beta(L+\bar{L})+\gamma_{u}\left(u^{c}+\bar{u}^{c}\right)+\gamma_{d}\left(d^{c}+\bar{d}^{c}\right)+h H
$$

In our six-stack models this matter content turns out to be independent of $n_{2}$. We find

$$
\alpha=3, \quad \beta=9, \quad \gamma_{u}=0=\gamma_{d}, \quad h=6
$$




\begin{tabular}{|c|c|c|c|}
\hline \hline Intersection & Multiplicity & Gonion & Mass $^{2}$ \\
\hline \hline$(12)$ & 3 & Fermions $Q_{L}+Q_{L}$ & $2 n|1-\delta| m_{H}^{2}$ \\
\hline$(13)$ & 3 & Fermions $u^{c}+\bar{u}^{c}$ & $2 n|\delta| m_{H}^{2}$ \\
\hline$(14)$ & 3 & Fermions $d^{c}+d^{c}$ & $2 n|\delta| m_{H}^{2}$ \\
\hline$(23)$ & 6 & Fermions $L+\bar{L}$ & $2 n m_{H}^{2}$ \\
& 6 & Scalars $H$ & $(2 n+1) m_{H}^{2}$ \\
& 6 & Vectors $H$ & $(2 n+1) m_{H}^{2}$ \\
\hline$(24)$ & 6 & Fermions $L+\bar{L}$ & $2 n m_{H}^{2}$ \\
& 6 & Scalars $H$ & $(2 n+1) m_{H}^{2}$ \\
& 6 & Vectors $H$ & $(2 n+1) m_{H}^{2}$ \\
\hline$(25)$ & 6 & Fermions $L+\bar{L}$ & $2 n|1-\delta| m_{H}^{2}$ \\
\hline$(26)$ & 3 & Fermions $L+L$ & $2 n|1-\delta| m_{H}^{2}$ \\
\hline$(45)$ & 6 & Fermions $e^{c}+\bar{e}^{c}$ & $2 n|\delta| m_{H}^{2}$ \\
\hline$(46)$ & 3 & Fermions $e^{c}+\bar{e}^{c}$ & $2 n|\delta| m_{H}^{2}$ \\
\hline \hline
\end{tabular}

Table 3: Gonion multiplicities and masses. $n$ is an integer.

The gauge group is that of the standard model apart from some extra $U(1)$ factors provided $n_{2} \neq 0 \bmod 3$. The weak hypercharge $Y$ is given by the superposition of $U(1)$ factors associated with the 6-stacks:

$$
-Y=\frac{1}{3} Q_{1}+\frac{1}{2} Q_{2}+Q_{3}+\left(Q_{5}^{(1)}+Q_{5}^{(2)}\right)+Q_{6}
$$

The mass of the tachyonic Higgs doublets [9] does depend on $n_{2}$ and is given by

$$
m_{H}^{2}=-\frac{m_{s}^{2}}{2 \pi} \frac{\epsilon\left(n_{2}-\delta\right)}{|\delta||1-\delta|}
$$

where $m_{s}$ is the string scale; $\epsilon$ and $\delta$ are related to the parameters defining the torus wrapped by the D4-branes. If $R_{1}$ and $R_{2}$ are the radii of the two fundamental 1-cycles, and $\theta$ is the angle between the two vectors defining the lattice, then

$$
\begin{array}{r}
\epsilon \equiv 2|\cos \theta / 2| \\
\delta \equiv n_{2}-3 R_{2} / R_{1}
\end{array}
$$

The above formula for $m_{H}^{2}$ is valid so long as $\epsilon \ll 1$, but in any case $m_{H}^{2} \ll m_{s}^{2}$ is required for consistency of the standard model without major contamination by string effects. $m_{H}^{2}$ also sets the scale for the various vector-like gonions that arise below the string scale. Specifically, we find the states whose masses and multiplicities are given in Table 3 .

The matter content detailed above contributes to the running of the coupling strengths $\alpha_{i}(\mu) \quad(i=3,2, Y)$ when the renormalization scale $\mu$ is greater than the 
mass of the relevant matter, and we can hence evaluate the coupling strengths at the (unknown) string scale $\mu=m_{s}$ at which their values are given in terms of the Type II string coupling $\lambda_{I I}$ and the wrapping numbers relevant to the stack producing the gauge group. We assume that the $\mathcal{N}=2$ gauge vector supermultiplet is massless over the entire range starting from $\mu=m_{Z}$. There are also Kaluza-Klein and winding modes partners of gauge bosons. The former will have masses above the string scale $m_{s}$ provided

$$
\frac{\alpha_{i}\left(m_{s}\right)}{\lambda_{I I}} \geq 1, \forall i
$$

The latter will have masses above $m_{s}$ if in addition

$$
\frac{4 \pi}{3}|\epsilon|\left(n_{2}-\delta\right)\left(m_{s} R_{1}\right)^{2} \geq 1
$$

We shall assume eqns (23) and (24) in what follows. In practice, we find values of $|\epsilon| \sim 10^{-1}$ so that Eq.(24) is satisfied when $R_{1} \geq m_{s}^{-1}$. Then, at the string scale the gauge couplings are given by:

$$
\begin{aligned}
\alpha_{3}^{-1}\left(m_{s}\right)= & \alpha_{3}^{-1}\left(m_{Z}\right)+\frac{1}{\pi} \ln \frac{m_{s}}{m_{Z}}-\frac{1}{\pi}\left(N_{3} \ln X_{3}-\ln N_{3} !\right) \\
& -\frac{1}{\pi}\left(N_{2} \ln X_{2}-\ln N_{2} !\right)
\end{aligned}
$$

where

$$
\begin{aligned}
& X_{3} \equiv\left|\frac{\delta \pi}{\epsilon\left(n_{2}-\delta\right)}\right| \quad \text { and } \quad N_{3} \equiv\left[X_{3}\right] \\
& X_{2} \equiv\left|\frac{(1-\delta) \pi}{\epsilon\left(n_{2}-\delta\right)}\right| \quad \text { and } \quad N_{2} \equiv\left[X_{2}\right]
\end{aligned}
$$

The integers $N_{2,3}$ count the numbers of colour triplet gonions with masses below $m_{s}$. In eqn (25), and eqns (28), (30) below, we have not included the effects of running the gonion and Higgs masses. We find that the gonion contributions to our solutions are small, so there is post hoc justification for our approximation.

$$
\begin{aligned}
\alpha_{2}^{-1}\left(m_{s}\right)= & \alpha_{2}^{-1}\left(m_{Z}\right)-\frac{6}{2 \pi} \ln \frac{m_{h}}{m_{Z}}-\frac{7}{2 \pi} \ln \frac{m_{s}}{m_{h}}-\frac{3}{\pi}\left(N_{3} \ln X_{3}-\ln N_{3} !\right) \\
& -\frac{2}{\pi}\left(N_{1} \ln X_{1}-\ln N_{1} !\right)-\frac{3}{4 \pi}\left[\left(N_{0}+1\right) \ln 2 X_{1}-\ln \frac{\left(2 N_{0}+1\right) !}{2^{N_{0}} N_{0} !}\right]
\end{aligned}
$$

where

$$
X_{1} \equiv\left|\frac{\delta(1-\delta) \pi}{\epsilon\left(n_{2}-\delta\right)}\right|, \quad N_{1} \equiv\left[X_{1}\right] \quad \text { and } \quad N_{0} \equiv\left[X_{1}-1 / 2\right]
$$


$N_{1}$ counts the number of lepton doublet gonions below the string scale, and $2 N_{0}+1$ is the number of scalar and vector gonions with masses below this scale.

$$
\begin{aligned}
\alpha_{Y}^{-1}\left(m_{s}\right) & =\alpha_{Y}^{-1}\left(m_{Z}\right)-\frac{50}{6 \pi} \ln \frac{m_{h}}{m_{Z}}-\frac{53}{6 \pi} \ln \frac{m_{s}}{m_{h}}-\frac{5}{3 \pi}\left(N_{3} \ln X_{3}-\ln N_{3} !\right) \\
-\frac{14}{3 \pi}\left(N_{2} \ln X_{2}-\ln N_{2} !\right) & -\frac{2}{\pi}\left(N_{1} \ln X_{1}-\ln N_{1} !\right) \\
& -\frac{3}{4 \pi}\left[\left(N_{0}+1\right) \ln 2 X_{1}-\ln \frac{\left(2 N_{0}+1\right) !}{2^{N_{0}} N_{0} !}\right]
\end{aligned}
$$

The ratios of the gauge coupling strengths, which depend on the length of the cycles $\left(n_{a}, m_{a}\right)$ and on the superposition of the $U(1)$ factors in the weak hypercharge [8], are independent of the unknown $\lambda_{I I}$ :

$$
\begin{aligned}
& \frac{\alpha_{2}^{-1}\left(m_{s}\right)}{\alpha_{3}^{-1}\left(m_{s}\right)}=\left[\delta^{2}+n_{2}\left(n_{2}-\delta\right) \epsilon^{2}\right]^{1 / 2} \\
& \frac{\alpha_{Y}^{-1}\left(m_{s}\right)}{\alpha_{3}^{-1}\left(m_{s}\right)}=\frac{10}{3}+\frac{\alpha_{2}^{-1}\left(m_{s}\right)}{2 \alpha_{3}^{-1}\left(m_{s}\right)}+\left[(1-\delta)^{2}+\left(n_{2}-1\right)\left(n_{2}-\delta\right) \epsilon^{2}\right]^{1 / 2}
\end{aligned}
$$

where $\epsilon$ and $\delta$ are defined in equations (21) and (22). Thus, by substituting the solutions (25),(28) and (30) of the renormalization group equations into (31) and (32), we obtain two constraint equations on $\epsilon$ and $\delta$.

We also considered the case when the $\mathcal{N}=2$ supersymmetric partners of the gauge fields acquire masses on the scale of $m_{s}$ or greater so they do not contribute to the renormalization group equations. In that case, the coefficient of the $\ln \frac{m_{\mathrm{s}}}{\mathrm{m}_{\mathrm{Z}}}$ in eqn. (25) is replaced by $\frac{7}{2 \pi}$, the coefficients of $\ln \frac{m_{h}}{m_{Z}}$ and $\ln \frac{m_{s}}{m_{h}}$ in eqn.(28) become $-\frac{4}{3 \pi}$ and $-\frac{11}{6 \pi}$ respectively, and in eqn.(30) become $-\frac{25}{3 \pi}$ and $-\frac{53}{6 \pi}$ respectively.

We solve the constraints (31) and (32) numerically for a range of values of the parameters $n_{2}$ and $m_{s}$, and using the latest values [12 of the standard model parameters which yield

$$
\begin{array}{r}
\alpha_{3}^{-1}\left(m_{Z}\right)=8.403 \\
\alpha_{2}^{-1}\left(m_{Z}\right)=29.776 \\
\alpha_{Y}^{-1}\left(m_{Z}\right)=99.124
\end{array}
$$

for the coupling strengths at the weak scale. For given values of the parameters $n_{2}$ and $m_{s}$, the calculated values of $\epsilon$ and $\delta$ determine the mass of the tachyonic Higgs doublets using equation (20), and this determines the mass $m_{h}$ of the physical Higgs particle from

$$
m_{h}^{2}=\left|2 m_{H}^{2}\right|
$$

The ratio $a$ of the compactification radii is then determined from eqn (22). Our results are summarised in Table 4 for the case of an $\mathcal{N}=2$ supersymmetric gauge sector, and in Table 5 when the $\mathcal{N}=2$ gauge superpartners are assumed to have masses of order $m_{s}$. 


\begin{tabular}{|c|c|c|cc|c|}
\hline \hline$n_{2}$ & $m_{s}(\mathrm{TeV})$ & $\epsilon$ & $m_{h}(\mathrm{GeV})$ & $a$ \\
\hline \hline 5 & 1.0 & 0.185 & 137 & 0.294 \\
\hline 5 & 1.2 & 0.182 & 165 & 0.297 \\
\hline 5 & 1.9 & 0.176 & 265 & 0.304 \\
\hline 5 & 3.0 & 0.170 & 425 & 0.312 \\
\hline \hline 10 & 1.0 & 0.123 & 137 & 0.198 \\
\hline 10 & 1.2 & 0.121 & 165 & 0.199 \\
\hline 10 & 1.9 & 0.116 & 265 & 0.202 \\
\hline 10 & 3.0 & 0.111 & 425 & 0.206 \\
\hline \hline 20 & 1.0 & 0.074 & 137 & 0.119 \\
\hline 20 & 1.2 & 0.073 & 165 & 0.120 \\
\hline 20 & 1.3 & 0.072 & 179 & 0.120 \\
\hline 20 & 1.9 & 0.069 & 265 & 0.121 \\
\hline 20 & 3.0 & 0.066 & 425 & 0.122 \\
\hline \hline
\end{tabular}

Table 4: Predicted values of the physical Higgs mass $m_{h} \equiv\left|2 m_{H}^{2}\right|^{1 / 2}$ and the ratio $a \equiv R_{1} / R_{2}$ of the compactification radii in the case of an $\mathcal{N}=2$ supersymmetric gauge sector.

We find that it is easy to obtain values of $\epsilon \ll 1$ and $m_{H}^{2} \ll m_{s}^{2}$ consistently with a string scale $m_{s}$ not more than a few TeV. Physical Higgs masses not much greater than the current LEP bound $\left(m_{h}>114 \mathrm{GeV}\right)$ [13] are found for some values of the parameters, but these are probably excluded because they are necessarily accompanied by charged, vector-like gonions with similar masses. The general behaviour is that with $m_{s}$ fixed, $m_{h}$ falls slowly as $n_{2}$ increases, whereas with $n_{2}$ fixed, $m_{h}$ increases as $m_{s}$ increases. As in [2, 3], with D-branes located at $R / Z_{3}$ orbifold fixed points, the mixed $U(1)$ anomalies in the present intersecting brane model are cancelled by a generalized Green-Schwarz mechanism mediated by twisted RamondRamond fields. However, it is unclear whether the anomalous $U(1)$ s survive as global symmetries because of the lack of supersymmetry in the present set-up. Certainly, the Yukawa terms needed to generate the required masses conserve all of the $U(1)$ symmetries, so it seems likely that they are global symmetries. If so, keV-scale axions will remain a problem in these models too. Unlike the models of [2], [3] , the present model possesses lepton mass terms as well as quark mass terms, at renormalizable level provided we assign the lepton doublets to (25) interesections rather than the (24) intersection. Also unlike these models our model is free from lepton number violating terms at renormalizable level. On the other hand it shares with those models the virtue of proton decay being perturbatively forbidden as a result of the selection rules discussed after (16) [9], although baryon number $B$ non-conservation with $\Delta B$ even is allowed. Masses for the vector-like matter are more problematic because of the lack 


\begin{tabular}{|c|c|c|c|c|}
\hline \hline$n_{2}$ & $m_{s}(\mathrm{TeV})$ & $\epsilon$ & $m_{h}(\mathrm{GeV})$ & $a$ \\
\hline \hline 5 & 1.0 & 0.130 & 145 & 0.345 \\
\hline 5 & 1.2 & 0.125 & 175 & 0.353 \\
\hline 5 & 1.9 & 0.112 & 286 & 0.370 \\
\hline 5 & 3.0 & 0.099 & 466 & 0.389 \\
\hline \hline 10 & 1.0 & 0.082 & 145 & 0.219 \\
\hline 10 & 1.2 & 0.078 & 175 & 0.222 \\
\hline 10 & 1.9 & 0.069 & 286 & 0.229 \\
\hline 10 & 3.0 & 0.06 & 466 & 0.236 \\
\hline \hline 20 & 1.2 & 0.045 & 175 & 0.128 \\
\hline 20 & 1.3 & 0.044 & 191 & 0.128 \\
\hline 20 & 1.9 & 0.039 & 286 & 0.130 \\
\hline 20 & 3.0 & 0.034 & 466 & 0.132 \\
\hline \hline
\end{tabular}

Table 5: Predicted values of the physical Higgs mass $m_{h} \equiv\left|2 m_{H}^{2}\right|^{1 / 2}$ and the ratio $a \equiv$ $R_{1} / R_{2}$ of the compactification radii in the case when the $\mathcal{N}=2$ gauge superpartners have masses of order $m_{s}$.

of gauge-singlet scalars amongst the tachyonic states to develop vacuum expectation values. Three of the $L+\bar{L}$ states and three of the $e_{L}^{c}+\bar{e}_{L}^{c}$ states acquire masses at tree-level via the usual $L e_{L}^{c} H$ and $\bar{L} \bar{e}_{L}^{c} H^{\dagger}$ couplings. The remaining six $L+\bar{L}$ pairs are able to acquire masses via non-renormalizable terms of the form $L \bar{L} H H^{\dagger}$; the latter are suppressed by a factor $\langle H\rangle / m_{s}$.

In conclusion, we have found a unique class of six-stack standard-like models that give masses to all of the matter, and are free of charged singlet (and colour triplet) tachyons. We find it relatively easy to ensure that the Higgs mass is small compared with the $1-3 \mathrm{TeV}$ string scale, while consistently reproducing the observed values of the gauge coupling constants at the electroweak scale, and achieving the values required by the string theory at the string scale. However, our models do have extra light, vector-like lepton states (but not quark states), and Higgs doublets, as well as the towers of gonions characteristic of all theories of this type. There are also three extra non-anomalous $U(1)$ gauge groups under which the matter is charged. 


\section{Acknowledgements}

This research is supported in part by PPARC. We are grateful to Angel Uranga for pointing out the error in the previous version of this paper concerning the generalized Green-Schwarz mechanism.

\section{References}

[1] G. Aldazabal, L. E. Ibáñez and F. Quevedo, JHEP 0001(2000)031, hepth/9909172; I. Antoniadis, E. Kiritsis and T. Tomaras, Phys.Lett.B486(2000)186, hep-th/0004214; R. Blumenhagen, L. Goerlich, B. Koers and D. Lüst, JHEP 0010(2000)006, hep-th/0007024; Z. Kakushadze, Phys.Rev. D59(1999)045007; Z. Kakushadze, G. Shiu and S.-H. Henry Tye, Nucl.Phys.B533(1998)25; J. Lykken, E. Poppitz, S. P. Trivedi, Nucl.Phys.B543(1999)105; G. Aldazabal and A. M. Uranga, JHEP 9910 (1999) 024, hep-th/9908072; I. Antoniadis, E. Dudas, A. Sagnotti, Phys.Lett.B 464 (1999)38; S. Sugimoto, Prog. Theor. Phys. 102(1999)685; C. Angelantonj, Nucl.Phys.B 566(2000)126;

[2] G. Aldazabal, L. E. Ibáñez, F. Quevedo and A. M. Uranga, JHEP 0008 (2000) 002, hep-th/0005067

[3] D. Bailin, G. V. Kraniotis and A. Love, Physics Letters B 502 (2001) 209, hepth/0011289

[4] We are grateful to Mark Hindmarsh for pointing this out.

[5] M. Berkooz, M. R. Douglas and R. G. Leigh, Nuclear Physics b480 (1996) 265, hep-th/9606139

[6] C. Bachas, A way to break supersymmetry, hep-th/9503030

[7] R. Blumenhagen, L. Görlich, B. Kors and D. Lüst, JHEP 0010 (2000) 006, hepth/0007024

[8] G. Aldazabal, S. Franco, L. E. Ibáñez, R. Rabadán and A. M. Uranga, hepth/0011073

[9] G. Aldazabal, S. Franco, L. E. Ibáñez, R. Rabadán and A. M. Uranga, JHEP 0102 (2001) 047, hep-ph/0011132

[10] C. Angelantonj, I. Antoniadis, E. Dudas and A. Sagnotti, Phys.Lett.B489(2000)223, hep-th/0007090;

R. Blumenhagen, B. Körs and D. Lüst, hep-th/0012156; L. E. Ibáñez, F. Marchesano and R. Rabadan, hep-th/0105155; S. Förste, G. Honecker and R. Schreyer, hep-th/0105208; R. Blumenhagen, B. Körs D. Lüst and T. Ott, hep-th/0107138; M. Cvetič, G. Shiu and A. M. Uranga, hep-th/0107166 
[11] G. Aldazabal, L. E. Ibáñez and F. Quevedo, JHEP 0002 (2000) 015, hepth/0005067

[12] Reports of the working groups on precision calculations for LEP2 Physics, S. Jadach, G. Passarino, R. Pittau (Eds.), CERN Yellow Report, CERN, 2000-009

[13] OPAL Collaboration, hep-ex/0107029 